\title{
Expression of Nitric Oxide Synthases and Nitrotyrosine during Blood-Brain Barrier Breakdown and Repair after Cold Injury
}

\author{
Sukriti Nag, Pierre Picard, and Duncan J. Stewart \\ The Toronto Western Research Institute (SN), University Health Network, Toronto, and Terrence Donnelly Heart \\ Center (PP, DJS), St. Michael's Hospital, University of Toronto, Toronto, Canada
}

\begin{abstract}
SUMMARY: This study was undertaken to determine whether the blood-brain barrier (BBB) breakdown and cerebral edema occurring post-trauma are associated with overexpression of the endothelial (e) and inducible (i) nitric oxide synthases (NOS), enzymes responsible for nitric oxide (NO) biosynthesis. These enzymes were determined quantitatively at the mRNA level and qualitatively at the protein level in the rat cerebral cortical cold injury model, during a period up to 6 days post-injury. In addition, peroxynitrite generation at the lesion site was detected by immunolocalization of nitrotyrosine as a marker of NO-superoxide interactions. These studies were correlated with the permeability status of the BBB by immunohistochemical detection of endogenous fibronectin extravasation in the same brains. BBB breakdown was immediate in lesion vessels, it was present as early as 10 minutes post-lesion and delayed in perilesional vessels that showed maximal BBB breakdown between 2-4 days. The BBB was restored to normal at 6 days post-lesion. An increase in both eNOS and iNOS mRNA was observed at the lesion site as compared with the contralateral hemisphere at 12 hours, 2 days, and 4 days. The mRNA returned to resting levels by 6 days. Increased eNOS protein was observed in the endothelium of permeable perilesional vessels and neovessels and in the endothelium of the hyperplastic pial vessels overlying the lesion site. iNOS protein was observed initially in polymorphonuclear leukocytes at the lesion site and later in macrophages, endothelial cells, and the smooth muscle cells of the overlying pial vessels. Furthermore, nitrotyrosine was demonstrated at the lesion site up to 5 days. Up-regulation of the NO synthases at both the mRNA and protein level accompanied by presence of nitrotyrosine during BBB breakdown and angiogenesis suggests that NO has a role in the pathogenesis of these processes. (Lab Invest 2001, 81:41-49).
\end{abstract}

$N$ itric oxide (NO) is produced from L-arginine and molecular oxygen by three isoforms of an NADPH-dependent enzyme, nitric oxide synthase (NOS) (Knowles and Moncada, 1994). Two isoforms of NOS are constitutively expressed in neurons and endothelial cells and are termed nNOS and eNOS, respectively. These isoforms are $\mathrm{Ca}^{2+}$-dependent and produce NO phasically in response to physiological stimuli. A third isoform of NOS, called inducible NOS (iNOS) is $\mathrm{Ca}^{2+}$-independent and is not constitutively expressed but requires cytokines or microbial product stimulation for induction of its expression (Billiar et al, 1990; Lorsbach et al, 1993; Nathan, 1992). Once expressed, iNOS produces large amounts of NO for prolonged periods of time (Xie and Nathan, 1994). The high output of NO favors the reaction between NO and the superoxide anion to form peroxynitrite (Beckman et al, 1994). The latter is a strong oxidant that can nitrate phenolic rings of tyrosine residues of proteins, leading to the formation of nitrotyrosine, which is easily detected with antinitrotyrosine antibodies (Beckman et al, 1994).

Received August 7, 2000.

Address reprint requests to: Dr. Sukriti Nag, Neuropathology, EW5-510, Toronto Western Hospital, 399 Bathurst Street, Toronto, Ontario M5T 2S8,Canada.E-mail:nag@uhnres.utoronto.ca
A few studies using NOS inhibitors suggest that NO may have a role in blood-brain barrier (BBB) breakdown. Decrease in BBB permeability to $\left[{ }^{14} \mathrm{C}\right]$ sucrose was observed after intravenous administration of aminoguanidine, an NOS inhibitor, to rats with meningitis produced by an intracisternal injection of lipopolysaccharide (Boje, 1996). The tracer $\left[{ }^{14} \mathrm{C}\right]$ sucrose is a sensitive marker of cellular damage because it has a radius of $5 \AA$. However, larger molecular weight substances such as fluorescein isothiocyanate dextran and Evans blue, which bind to albumin in vivo, may be better suited for studies of BBB permeability to protein that is most relevant to cerebral edema. Topical application of the NOS inhibitors L-NAME or L-NMMA on pial vessels resulted in reduced permeability of pial veins to fluorescent-labeled albumin in phenylephrineinduced acute hypertension (Mayhan, 1995). Although the permeability of pial endothelium to protein is not as restricted as that of the endothelium of intracerebral vessels, these findings suggest that NO has a role in BBB breakdown in pathological states.

The rat cortical cold injury model is an established and highly reproducible model of BBB breakdown and cerebral angiogenesis, and the time course of these processes is well defined (Nag, 1996; Nag et al, 1997). This model was used to determine the expression and cellular distribution of eNOS and iNOS during the 6 day period after cold injury. These isoforms were 
selected because both eNOS and iNOS have been demonstrated in cerebral endothelium in normal (Catalán et al, 1996) and pathological states, respectively, and suggest that NO may have a role in BBB homeostasis. Neuronal NOS was not studied, because a previous report demonstrated this isoform in endothelium of pial but not intracerebral vessels (Stanarius et al, 1998).

Gene expression of the NOS enzymes was quantitated by the ribonuclease protection assay, and protein was localized by immunohistochemistry. In addition, peroxynitrite generation at the lesion site was detected by immunolocalization of nitrotyrosine. The latter studies were correlated with the permeability status of the BBB by immunohistochemical detection of endogenous fibronectin extravasation in the same brains.

\section{Results}

\section{Morphology of the Cold Lesion}

The morphology of the cortical cold injury and the time course of the inflammatory and BBB changes were similar to our previous observations (Nag, 1996; Nag et al, 1997). At 10 minutes, the injury site showed a central area of coagulative necrosis, which extended from the pial surface into the 4th cortical layer. Neurons within the lesion showed nuclear pyknosis and shrunken cytoplasm, and neuronal loss was present within the lesion in two of three rats sacrificed at 6 hours and in all rats sacrificed at 12 hours. The cellular response was consistent, and an initial polymorphonuclear leukocyte response was followed by a macrophage response. The latter persisted up to the 6 day period of observation. The onset of angiogenesis was marked by an increase in the number of endothelial cells, which surrounded pre-existing vessels in the lesion starting from Day 2 (Fig. 1). At Day 4, profiles of neovessels were observed at the lesion site, and by Day 6 the entire lesion area showed profiles of neovessels. A consistent finding was the presence of mural thickening of the pial arterioles overlying the cold lesion site, and this was caused by hyperplasia of the medial smooth muscle cells and an increase in the extracellular matrix in this layer.

\section{BBB Breakdown}

Control Rats. Brains of control rats did not show BBB breakdown to endogenous fibronectin. Occasional poorly perfused vessels showed marked fibronectin immunoreactivity localized to the plasma proteins present in vessel lumina and served as a positive control for this protein.

Cold Injured Rats. The time course of BBB breakdown to endogenous fibronectin was similar to our previous observations (Nag et al, 1997). BBB breakdown was immediate, and at 10 minutes fibronectin extravasation was already noted from lesion vessels (Fig. 1). At 60 minutes, fibronectin extravasation from occasional perilesional vessels was present. At 12 hours, fibronectin was present throughout the lesion site and in continuity in the underlying white matter, the corpus callosum, and the white matter of the contralateral hemisphere. At 2 days, most perilesional vessels showed fibronectin in their walls and in continuity in the surrounding neuropil (Fig. 2, a and b). At 4 days, BBB breakdown was localized to the lesion site and marked fibronectin immunostaining was present extracellularly around the newly formed endothelial cells forming a mesh-like pattern. At 6 days, fibronectin immunostaining was confined to the extracellular

\section{Temporal and Spatial Protein Localizations at the Cold-Injury Site}

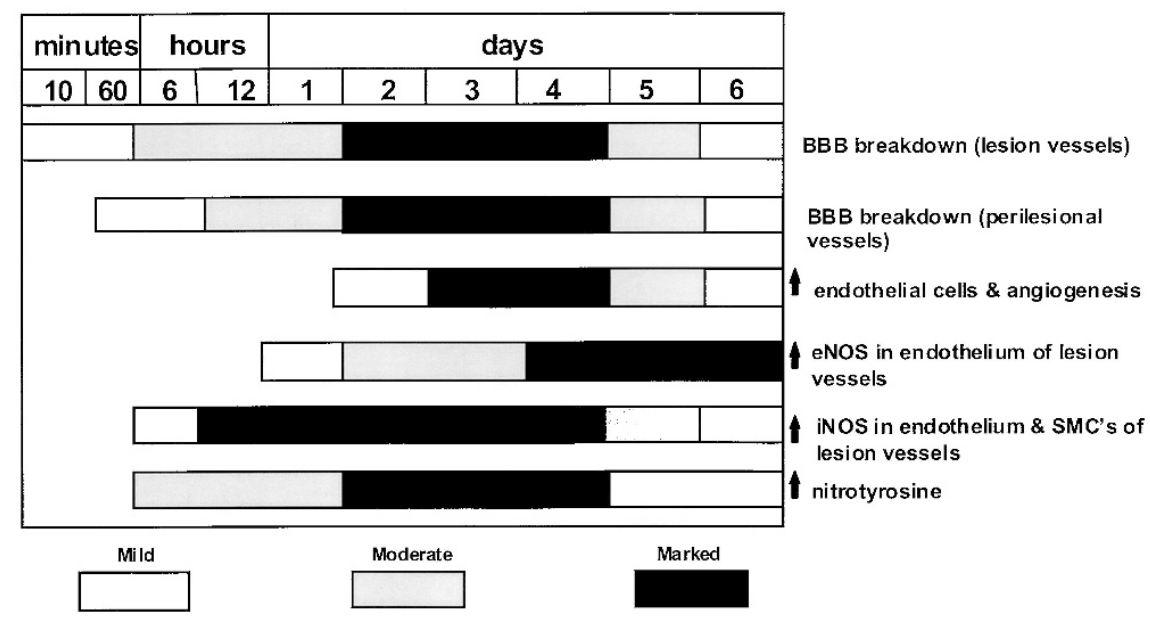

Figure 1.

Temporal and spatial localization of endothelial nitric oxide synthase (eNOS), inducible nitric oxide synthase (iNOS), and nitrotyrosine are shown in relation to blood-brain barrier (BBB) breakdown to endogenous fibronectin and angiogenesis at the cold injury site from 10 minutes to 6 days. 

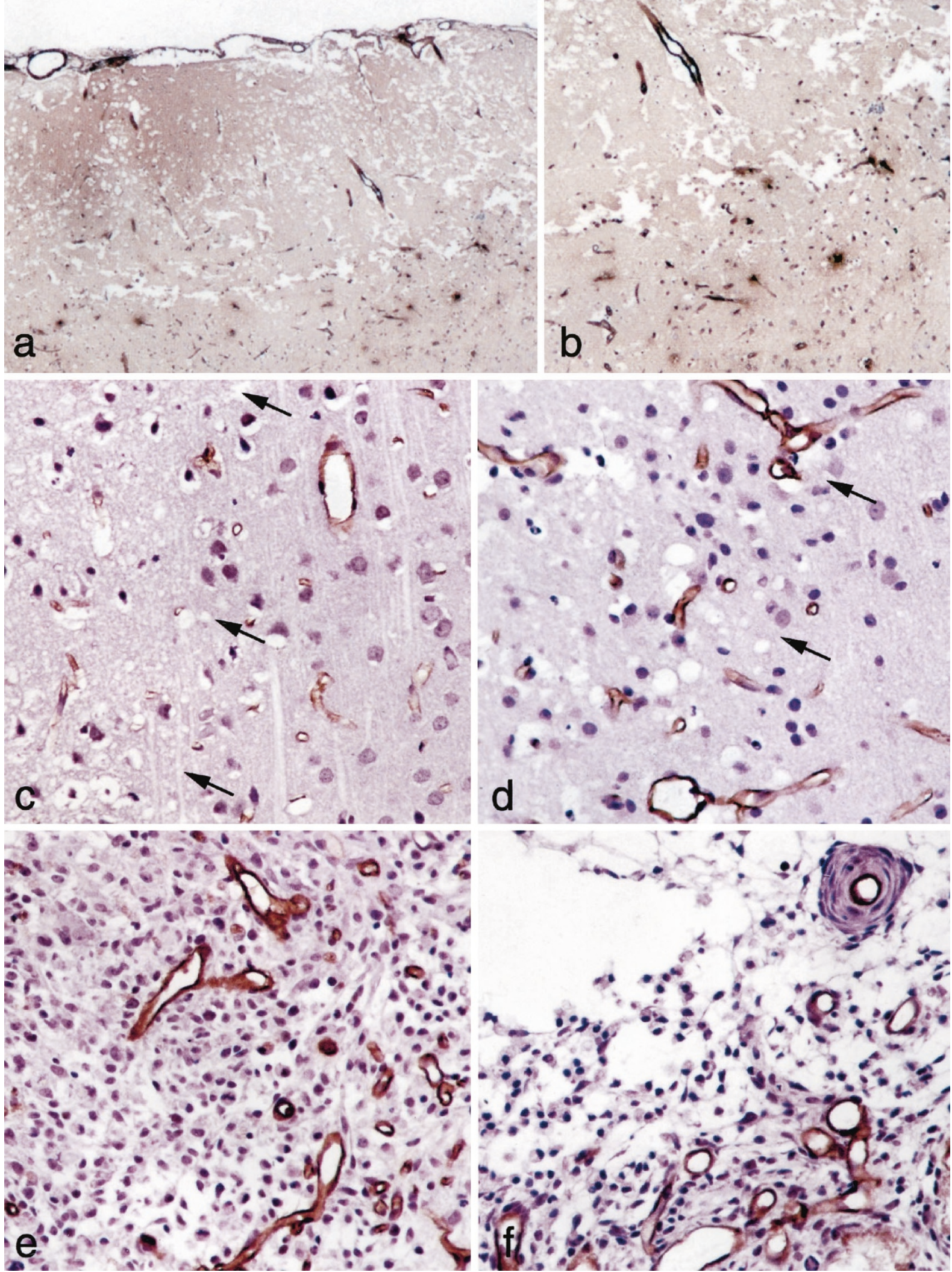

\section{Figure 2.}

Cold injury. Immunoreactivity for fibronectin (a and b) and eNOS ( $c$ to $f$ ) at the cold injury site is shown. a, Extravasation of endogenous fibronectin from lesion and perilesional vessels in the cold injury site at 2 days is shown. b. High magnification of perilesional vessels showing BBB breakdown to endogenous fibronectin. C, At 12 hours, mild eNOS immunoreactivity $(1+)$ is present in endothelium of lesion vessels and in vessels in the surrounding brain. The margin of the lesion and surrounding brain is marked by arrows in $\mathrm{c}$ and $\mathrm{d}$. At 2 days (d), there is a moderate $(2+)$ increase in eNOS immunoreactivity in perilesional vessels, which appear dilated and tortuous. e, At day 4, perilesional vessels and profiles of neovessels show marked eNOS immunoreactivity (3+). f, At day 6, the lesion site is occupied by neovessel profiles that show moderate-to-marked eNOS immunoreactivity. The pial artery with marked medial hyperplasia overlying the lesion shows marked endothelial eNOS immunoreactivity. Magnification: $a, \times 30 ; b, \times 130$; c to f, $\times 250$. 
matrix in walls of neovessels in the lesion site and the pial vessels overlying the lesion site.

\section{Expression of eNOS and iNOS mRNA}

The lesion site and the corresponding area of the contralateral hemisphere of test and control rats were assayed for eNOS, iNOS, and $\beta$-actin mRNA content by the ribonuclease protection assay. Compared with the contralateral hemisphere, significant up-regulation of eNOS mRNA levels was present at the lesion site at 2 and 4 days, the mean mRNA levels and the SEM were $2.2 \pm 0.4$-fold $(p<0.01)$ and $1.2 \pm 0.2$-fold $(p<0.01)$ over control values, respectively. In the case of iNOS, significant up-regulation of mRNA levels was present in the lesion site of test rats as compared with the contralateral nonlesioned hemisphere at 12 hours, 2 days, and 4 days. The mean iNOS mRNA values for these time periods were $5.4 \pm 0.8$-fold $(p<0.01), 5.5$ \pm 0.7 -fold $(p<0.05)$, and $3.6 \pm 0.7$-fold $(p<0.05)$, respectively, over control values (Fig. $3 b$ ). In addition, the iNOS mRNA values in the nonlesioned hemisphere were significantly different from those of controls at 12 hours and 2 days, with the increase being $2.7 \pm$ 0.2-fold and $2.6 \pm 0.2$-fold, respectively, over control values. At 6 days, the mean eNOS and iNOS mRNA values at the lesion site were $1.0 \pm 0.1$-fold and $1.7 \pm$ 0.4-fold, respectively, values that were similar to those obtained from corresponding areas of the contralateral hemisphere for this time period.

\section{NOS Immunoreactivity}

Endothelial NOS. The endothelium of all vessels throughout the brain of control rats showed mild eNOS immunoreactivity. eNOS immunoreactivity was not observed in other cell types such as astrocytes or neurons of control rats. At 12 hours, eNOS immunoreactivity in vessels in the cold injury site was similar to that of vessels in the surrounding neuropil (Fig. 2c). However, fewer microvessel profiles were present as compared with the contralateral cortex. eNOS immunoreactivity in endothelium of perilesional vessels was moderate at 2 days (Fig. 2d) and marked at 4 days (Figs. 1 and 2e). Proliferating endothelial cells failed to show eNOS immunostaining, however, once vascular tubes were formed they were lined by endothelium, which showed marked eNOS immunostaining (Fig. 2, e and f). At 6 days, the endothelium of pial vessels overlying the lesion site also showed marked eNOS immunoreactivity (Fig. 2f). eNOS immunoreactivity was not observed in the increased astrocytes present in the perilesional area or in those present in the underlying white matter.

Inducible NOS. Brains of control rats failed to show iNOS immunoreactivity. Cold injured rats showed mild endothelial iNOS immunoreactivity in pial vessels overlying the lesion site at 6 hours (Fig. 1). At 12 hours, marked immunoreactivity was observed in the endothelium of these vessels and in the cytoplasm of marginating polymorphonuclear leukocytes and those present extravascularly in the neuropil (Fig. 4a). At 2 a

\section{eNOS mRNA Expression}

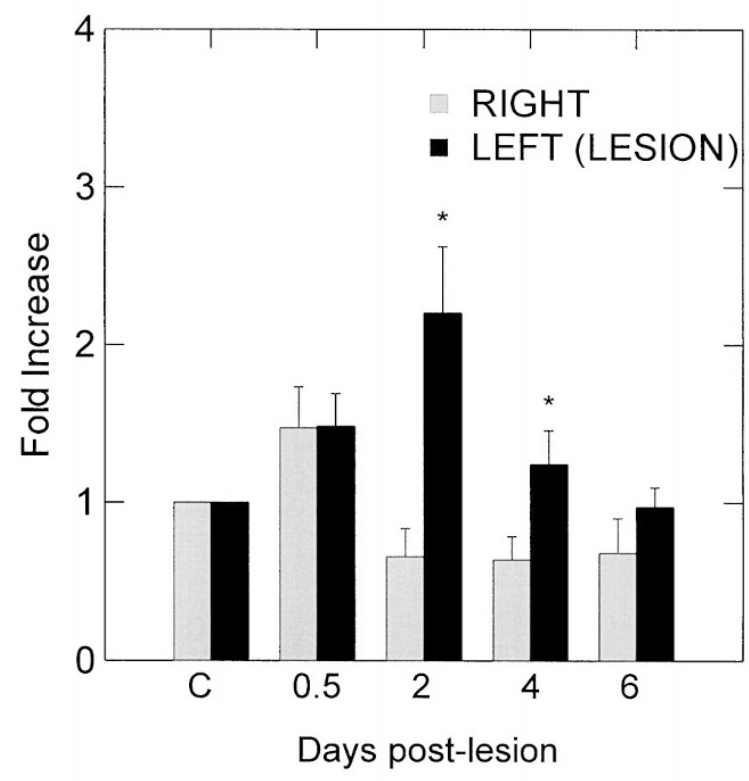

b

\section{iNOS mRNA Expression}

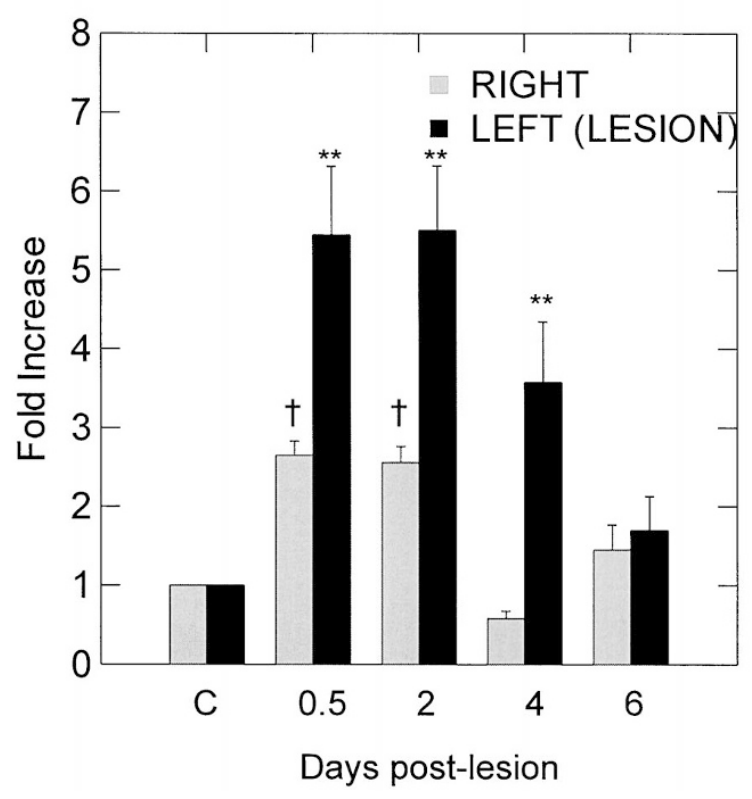

Figure 3.

eNOS (a) and INOS (b) mRNA expression relative to $\beta$-actin at the cold injury site (left) and the cortex of the contralateral hemisphere (right) at 0.5, 2, 4, and 6 days are shown. Values represent means \pm SEM. a, There is a significant increase in eNOS mRNA at the lesion site at 2 and 4 days. b, In the case of iNOS, a significant increase in MRNA is present at the lesion site at 12 hours and at 2 and 4 days. Also present is a significant increase in iNOS mRNA expression in the nonlesioned hemisphere of test rats as compared with controls at 0.5 and 2 days. At each time point, $n=5$. Asterisks denote statistical significance as compared with the corresponding area of the contralateral hemisphere $\left({ }^{* *} p<0.01 ;{ }^{*} p<0.05\right)$. Crosses depict statistical significance in the nonlesioned hemisphere of test rats as compared with the ipsilateral hemisphere of controls $(\dagger p<0.05)$. 
days marked iNOS immunoreactivity was observed in the endothelium of perilesional vessels and in macrophages and residual polymorphonuclear leukocytes at the lesion site (Fig. 4b). Macrophages were readily recognized by their oval eccentric nuclei and foamy cytoplasm. An additional finding at 4 days was marked iNOS immunoreactivity in the proliferating endothelial cells at the lesion site (Fig. 4c). At 6 days, mild iNOS immunoreactivity was confined to the smooth muscle cells of the pial vessels overlying the lesion (Fig. 4d).

\section{Nitrotyrosine}

Nitrotyrosine immunoreactivity was localized to the lesion site and first observed at 3 hours when marked immunostaining was present in the neuropil, which appeared vacuolated and in neuronal cytoplasm (Fig. 4 e). Moderate and then mild nitrotyrosine immunoreactivity in the neuropil persisted up to 4 days. At 6 hours, residual neurons were not present in the lesion site and cytoplasmic nitrotyrosine immunoreactivity was present in polymorphonuclear leukocytes present intravascularly and those present extravascularly in the neuropil. Macrophages in the lesion area showed marked cytoplasmic immunoreactivity for nitrotyrosine up to 4 days, after which mild immunostaining of approximately $25 \%$ of macrophages was observed up to 6 days (Fig. 4f).

\section{Discussion}

This study demonstrates up-regulation of both eNOS and iNOS after brain injury. These changes are associated with evidence of BBB breakdown and nitrotyrosine at the lesion site.

\section{The Cortical Cold Injury Model}

This model is widely used to study the pathogenesis of BBB breakdown and the inflammatory response posttrauma (Grzybicki et al, 1998; Knerlich et al, 1999; Nag, 1996; Nag et al, 1997). However, the technique for production of the cold lesion is not uniform in all studies. Some groups place the cold probe on the intact skull (Grzybicki et al, 1998), whereas others place it on the dura after a craniotomy (Knerlich et al, 1999; Nag et al, 1997). There is also a variation in the diameter of the cold probe used and the total time period that the probe is placed on the skull. These differences could account for the variation in the size and the histology of the lesions post-trauma. Only a few authors have studied the late tissue responses after a cold injury and observed the angiogenesis that occurs in this model (Cancilla et al, 1979; Olson et al, 1987). The techniques used in this study result in a consistent angiogenic response, and the time course of cellular events is similar but not identical to that reported in the mouse cold injury model (Cancilla et al, 1979). The associated cell types present during angiogenesis have been characterized in our previous studies by immunolocalization of glucose transporter-1 protein in endothelial cells and glial-fibrillary acidic protein in astrocytes, whereas polymorphonuclear leukocytes and macrophages were recognized by their characteristic morphology (Nag, 1996; Nag et al, 1997).

\section{BBB Breakdown and Angiogenesis}

The time period of observed BBB breakdown to fibronectin, from 10 minutes to 5 days post-injury, is similar to our previous observations (Nag, 1996; Nag et al, 1997). Immediate BBB breakdown after production of the cold lesion is caused by mechanical destruction of vessels by the cold temperature, and is unrelated to the effects of permeability mediators. Of importance is the subsequent BBB breakdown of lesion and perilesional vessels, which is associated with the effects of mediators such as bradykinin (Maier-Hauff et al, 1984), oxygen radicals (Ando et al, 1989), and vascular endothelial growth factor (VEGF) (Nag et al, 1997). The onset of BBB breakdown preceded angiogenesis and this is in keeping with the role of vascular hyperpermeability in promoting angiogenesis by providing a protein matrix that facilitates the migration of endothelial cells (Dvorak et al, 1995; Nag et al, 1997).

\section{Overexpression of eNOS}

Up-regulation of eNOS mRNA occurred at the lesion site at 12 hours, with significant increases over baseline levels occurring at 2 and 4 days. There are no previous studies of eNOS mRNA in brain post-trauma. Increased eNOS protein was observed in endothelium of perilesional vessels at 2 days and later in endothelium of the neovessels present within the lesion and in the endothelium of pial vessels overlying the lesion site. One other study shows increased eNOS protein in endothelium of lesion vessels up to 48 hours after trauma (Gahm et al, 2000). However, presence of eNOS protein was not correlated with BBB breakdown or angiogenesis, and eNOS localization in neovessels was not reported.

Studies of eNOS knockout mice demonstrate that eNOS has a protective effect in the brain. After cerebral ischemia, eNOS deficient mice have larger cerebral infarcts than the wild-type mice, and this effect is attributed to the beneficial hemodynamic effects of NO (Huang et al, 1996). Another known beneficial effect of eNOS, which is relevant to the present study, is its role in vascular remodeling and angiogenesis because angiogenesis in the ischemic hindlimb is significantly impaired in eNOS -/- mice versus wildtype controls (Rudic et al, 1998; Murohara et al, 1998).

Our previous studies demonstrated increased immunolocalization of VEGF protein during the period of BBB breakdown and angiogenesis in the cold injury model (Nag et al, 1997). The interaction of VEGF and NOS has been studied in noncerebral vessels, and results indicate that eNOS acts downstream from VEGF (Rudic et al, 1998; Ziche et al, 1997) and that its expression in native and cultured endothelial cells is enhanced by VEGF (Bouloumié et al, 1999). 

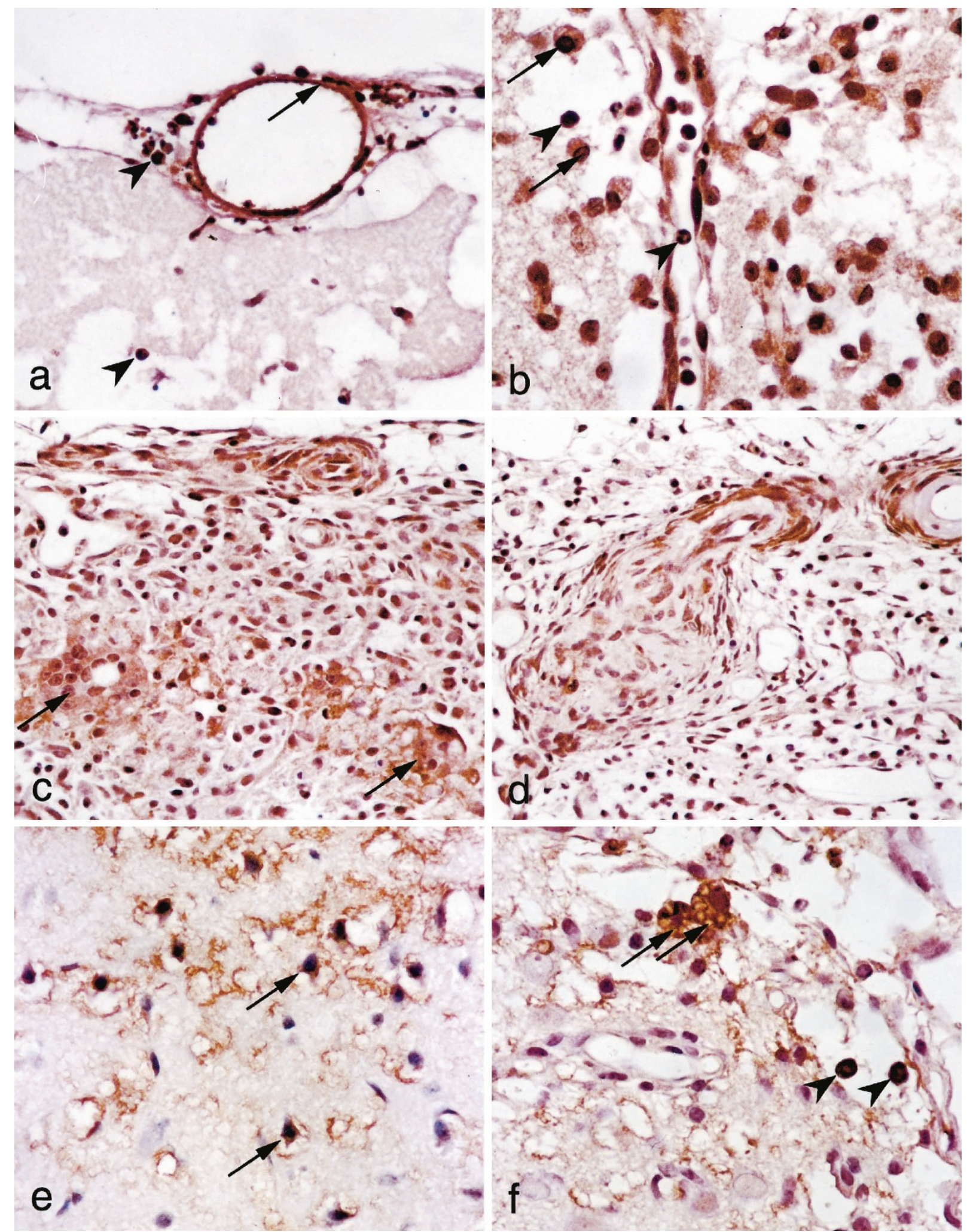
(b)

Figure 4.

iNOS and nitrotyrosine immunoreactivity at the cold injury site. iNOS: At 12 hours the cold injury site shows necrosis in the neuropil (a). Marked iNOS immunoreactivity is present in polymorphonuclear leukocytes (arrowheads) and in the endothelium of the overlying pial vein (arrow). b, At day 2, iNOS immunoreactivity is present in the endothelium of an intracerebral venule, in polymorphonuclear leukocytes (arrowheads), and in most macrophages present (arrows). c. At day 4, iNOS immunoreactivity is present in the multinucleate endothelial cells at the lesion site (arrows) and in the endothelium and smooth muscle cells of the pial artery overlying the lesion. $d$, At day 6 , mild to moderate $(1+$ to $2+)$ iNOS immunoreactivity is present in the hyperplastic smooth muscle cells of the pial arteries overlying the lesion site. e, At 3 hours, cytoplasmic nitrotyrosine immunoreactivity is present in neurons within the lesion and in the surrounding extracellular spaces. $f$, At 4 days, cytoplasmic nitrotyrosine immunoreactivity is present in macrophages (arrows) and polymorphonuclear leukocytes (arrowheads). The neuropil shows diffuse nitrotyrosine immunoreactivity. Magnification: $a, b, e$, and $f, \times 500 ; c$ and $d, \times 250$. 


\section{Overexpression of iNOS}

Up-regulation of iNOS mRNA in this study was widespread; it was present at the lesion site at 12 hours, 2 days, and 4 days and in the contralateral nonlesioned hemisphere at 12 hours and 2 days. Several studies have documented an up-regulation of iNOS mRNA in diverse pathologies such as stab injury and CNS inflammation (Cross et al, 1996; Garcion et al, 1998), cerebral ischemia (ladecola, 1997), and trauma (Grzybicki et al 1998; Knerlich et al, 1999). However, the time course and the total time period of iNOS upregulation varies in the different models, and is shortest after severe injury, as seen after focal cerebral ischemia (ladecola, 1997).

In this study, iNOS protein was first observed in polymorphonuclear leukocytes and later in endothelial cells, vascular smooth muscle cells, and macrophages. Several studies of various types of brain trauma have reported the presence of iNOS protein in a variety of cell types such as microglia (Knerlich et al, 1999), polymorphonuclear leukocytes (Clark et al, 1996; Gahm et al 2000), astrocytes (Grzybicki et al 1998; Wada et al, 1998), vascular smooth muscle cells (Clark et al, 1996), and macrophages (Gahm et al 2000; Wada et al, 1998). In these studies there are variations in the time course of the inflammatory response and the cell types showing iNOS protein. None of these studies reported presence of iNOS protein in endothelial cells as described in the present study.

Data from iNOS deficient mice indicate that iNOS expression is associated with a deleterious effect in tissues because these mice have smaller infarcts than wild-type mice after cerebral ischemia (ladecola, 1997). Up-regulation of iNOS was greater and more sustained than eNOS indicating a dominating deleterious effect of iNOS in this model, a finding supported by the nitrotyrosine data.

Overexpression of eNOS and iNOS at the mRNA and protein level during BBB breakdown and angiogenesis suggests that $\mathrm{NO}$ has a role in these processes. The biological actions of $\mathrm{NO}$ are complex and appear to be dose-dependent (ladecola, 1997), therefore its precise role in BBB breakdown and angiogenesis in this model remains speculative. However, based on its known functions, NO may induce vasodilatation and BBB breakdown before angiogenesis. Other studies have implicated NO in the BBB breakdown produced by lipopolysaccharides (Boje, 1996). Nitric oxide is also known to have a growth inhibitory effect (Babaei et al, 1998); therefore it may reduce the initial proliferative response during the early phase of angiogenesis, which is characterized by endothelial cell activation. However, during the later stages of angiogenesis, NO may initiate a program of endothelial cell differentiation and capillary tube formation as observed in noncerebral vessels (Babaei et al, 1998; Papapetropoulos et al, 1997).

Although, this study demonstrates up-regulation of only eNOS and iNOS during BBB breakdown and angiogenesis, in vitro studies of noncerebral vessels have demonstrated up-regulation of all three isoforms of NOS during angiogenesis (Papapetropoulos et al, 1997).

\section{Nitrotyrosine}

Nitrotyrosine immunoreactivity was localized to the lesion site and observed in cortical neurons and in the surrounding neuropil at 3 hours. Later nitrotyrosine immunoreactivity was observed in polymorphonuclear leukocytes and macrophages as well as in the neuropil. Nitrotyrosine is derived from peroxynitrite, which is formed by the interaction of NO and the superoxide anion radical. Peroxynitrite is implicated in the pathogenesis of neurotoxicity in neurodegenerative diseases (Torreilles et al, 1999) and CNS inflammation (van der Veen et al, 1997). The presence of nitrotyrosine at the lesion site supports the hypothesis that overexpression of NOS in this model is associated with NO release. The finding of nitrotyrosine in neurons at the lesion site at 3 hours suggests that NO and superoxide may have contributed to neuronal death, because no neurons were demonstrable at the lesion site after this time point.

In summary, this study provides evidence implicating $\mathrm{NO}$ in BBB breakdown and angiogenesis after brain trauma. Further studies using the NOS null mice may provide data on the precise role of $\mathrm{NO}$ in the repair processes after brain trauma. The latter approach may be more suitable than the use of pharmacologic NOS inhibitors, which fail to selectively block individual NOS isoforms.

\section{Materials and Methods}

A cortical cold injury was produced in male Wistar rats (180-200 grams) as described previously (Nag, 1996). The protocol for animal experimentation was drawn up in accordance with guidelines established by the Canadian Council on Animal Care and approved by the local animal care committee. Rats were anesthetized by methoxyflurane inhalation. After a skin incision, a hole was made in the left parietal bone using a dental drill with a bit measuring $2.3 \mathrm{~mm}$ in diameter. A cold probe consisting of the tip of a copper wire, contained in a $20 \mathrm{ml}$ syringe containing liquid nitrogen, was placed over the brain through the craniotomy site for 45 seconds. The skin incision was sutured and rats were allowed to recover.

\section{mRNA Studies}

Rats were killed by decapitation in groups of 5 at 12 hours and 2, 4, and 6 days post-lesion. The cold injury site and the corresponding area from the contralateral cortex were excised and rapidly frozen. The standard guanidinium-isothiocyanate phenol-extraction procedure was used for RNA extraction as described previously (Chomczynski and Sacchi, 1987). eNOS, iNOS, and $\beta$-actin mRNA levels were quantitated using the ribonuclease protection assay kit (Ambion Inc., Austin, Texas). Biotinylated probes for eNOS (150 base pair fragment), iNOS (288 base pair fragment), or $\beta$-actin 
(313 base pair fragment) were hybridized with $10 \mu \mathrm{g}$ of total RNA extracted from each sample as described previously (Picard et al, 1998). A low molecular weight RNA ladder (0.15-1.7kb; Gibco BRL, Burlington, Otario, Canada) incorporated in every gel enabled accurate identification of the expected bands at 150 , 288 , and 313 bases, for eNOS, iNOS, and $\beta$-actin, respectively. The intensity of eNOS and iNOS bands was quantitated by densitometry (GS-700, BioRad, Mississauga, Ontario, Canada) and normalized to the $\beta$-actin density. Fold increase was calculated as the ratio of the experimental value over the mean of the control values.

\section{Statistical Analysis}

The optical density of bands after normalization to $\beta$-actin of each hemisphere of test and control rats killed at the different time periods were compared using ANOVA with a Tukey HSD post-hoc test using Systat 8.0 (Systat Inc., Evanston, Illinois). The mean optical density of bands from the lesion site was compared with that of the nonlesioned cortex of the different experimental groups using the paired $t$ test with the Bonferroni post-hoc test. Differences were considered significant at a value of $p<0.05$

\section{Immunohistochemical Studies}

Rats were killed in groups of 3 , along with 3 controls, at 10 and 60 minutes; 3, 6, and 12 hours; and 1, 2, 3, 4,5 , and 6 days. At the time of death, rats were anesthetized with Metofane and perfused with 3\% paraformaldehyde in $0.1 \mathrm{M}$ phosphate buffer as described previously (Nag, 1996). A coronal slab of brain containing the cold injury site was processed for paraffin sectioning using standard techniques. Sections $(5 \mu \mathrm{m})$ were stained with hematoxylin and eosin for histological analysis, and adjacent sections were used for immunohistochemistry.

The indirect streptavidin-biotin peroxidase method was used and paraffin sections were pretreated with $0.5 \%$ pepsin for 30 minutes at $37^{\circ} \mathrm{C}$ before overnight incubation in primary antibody at $4^{\circ} \mathrm{C}$. Dilutions of the rabbit antibodies used were: eNOS (Transduction Laboratories, Lexington, Kentucky) 1:50; iNOS (Santa Cruz Biotechnology Inc., Santa Cruz, California) 1:1200; fibronectin (Gibco BRL) 1:700; and Nitrotyrosine (Upstate Biotechnology, New York) 1:70. In the case of eNOS, immunostaining was enhanced by tyramide signal amplification using the protocol provided by the supplier (NEN Life Science Products, Boston, Massachusetts). The intensity of the immunostaining was assessed semiquantitatively in a blinded manner by assigning the following scores: -, no change; $1+$, mild increase; $2+$, moderate increase; $3+$, marked increase. This scoring was used to prepare Figure 1.

The controls for immunohistochemistry included omission of the primary antibody and the use of nonimmune serum in place of the primary antibody. In both cases immunoreactivity for eNOS, iNOS, and nitrotyrosine was abolished. In the case of nitrotyrosine, primary antibody was preadsorbed with $1 \mathrm{~mm}$ nitrotyrosine for 30 minutes before immunostaining. This abolished immunoreactivity for nitrotyrosine.

\section{Acknowledgements}

Thanks are expressed to Dan Kilty, Inge Frohn, and Huimin Wang for skilled technical assistance and to Valerie Butcher and Nima Nourhaghighi for assistance with the illustrations. This work was supported by the Ontario Neurotrauma Foundation.

\section{References}

Ando Y, Inoue M, Hirota M, Morino Y, and Araki S (1989). Effect of a superoxide dismutase derivative on cold-induced brain edema. Brain Res 477:286-289.

Babaei S, Teichert-Kuliszewska K, Monge J-C, Mohamed F, Bendeck MP, and Stewart DJ (1998). Role of nitric oxide in the angiogenic response in vitro to basic fibroblast growth factor. Circ Res 82:1007-1015.

Beckman JS, Ye YZ, Anderson PG, Chen J, Accavitti MA, Tarpey MM, and White CR (1994). Extensive nitration of protein tyrosines in human atherosclerosis detected by immunohistochemistry. Biol Chem Hoppe-Seyler 375:81-88.

Billiar TR, Curran RD, Ferrari FK, Williams DL, and Simmons RL (1990). Kupffer cell: Hepatocyte cocultures release nitric oxide in response to bacterial endotoxin. J Surg Res 48:349353.

Boje KMK (1996). Inhibition of nitric oxide synthase attenuates blood-brain barrier disruption during experimental meningitis. Brain Res 720:75-83.

Bouloumié A, Schini-Kerth VB, and Busse R (1999). Vascular endothelial growth factor up-regulates nitric oxide synthase expression in endothelial cells. Cardiovasc Res 41:773-780.

Cancilla PA, Frommes SP, Kahn LE, and De Bault LE (1979). Regeneration of cerebral microvessels. A morphologic and histochemical study after local freeze-injury. Lab Invest 40: 74-82.

Catalán RE, Martínez AM, Aragonés MD, and Hernández $F$ (1996). Identification of nitric oxide synthases in isolated bovine brain vessels. Neurosci Res 25:195-199.

Chomczynski P and Sacchi N (1987). Single-step method of RNA isolation by acid guanidinium thiocyanate-phenolchloroform extraction. Anal Biochem 162:156-159.

Clark RSB, Kochanek PM, Schwarz MA, Schiding JK, Turner DS, Chen M, Carlos TM, and Watkins SC (1996). Inducible nitric oxide synthase expression in cerebrovascular smooth muscle and neutrophils after traumatic brain injury in immature rats. Pediatr Res 39:784-790.

Cross AH, Keeling RM, Goorha S, San M, Rodi C, Wyatt PS, Manning PT, and Misko TP (1996). Inducible nitric oxide synthase gene expression and enzyme activity correlate with disease activity in murine experimental autoimmune encephalomyelitis. J Neuroimmunol 71:145-153.

Dvorak HF, Brown LF, Detmar M, and Dvorak AM (1995). Vascular permeability factor/vascular endothelial growth factor, microvascular hyperpermeability, and angiogenesis. Am J Pathol 146:1029-1039. 
Gahm C, Holmin S, and Mathiesen T (2000). Temporal profiles and cellular sources of the three nitric oxide synthase isoforms in the brain after experimental contusion. Neurosurgery 46:169-177.

Garcion E, Sindji L, Montero-Menei C, Andre C, Brachet P, and Darcy $F$ (1998). Expression of inducible nitric oxide synthase during rat brain inflammation: Regulation by $1,25-$ Dihydroxyvitamin D3. Glia 22:282-294.

Grzybicki D, Moore SA, Schelper R, Glabinski AR, Ransohoff RM, and Murphy $S$ (1998). Expression of monocyte chemoattractant protein (MCP-1) and nitric oxide synthase-2 following cerebral trauma. Acta Neuropathol 95:98-103.

Huang Z, Huang PL, Ma J, Meng W, Ayata C, Fishman MC, and Moskowitz MA (1996). Enlarged infarcts in endothelial nitric oxide synthase knockout mice are attenuated by nitroL-arginine. J Cereb Blood Flow Metab 16:981-987.

ladecola C (1997). Bright and dark sides of nitric oxide in ischemic brain injury. Trends Neurosci 20:132-139.

Knerlich F, Schilling L, Gorlach C, Wahl M, Ehrenreich H, and Siren AL (1999). Temporal profile of expression and cellular localization of inducible nitric oxide synthase, interleukin-1 beta and interleukin converting enzyme after cryogenic lesion of the rat parietal cortex. Mol Brain Res 68:73-87.

Knowles RG and Moncada S (1994). Nitric oxide synthases in mammals. Biochem J 98:249-258.

Lorsbach RB, Murphy WJ, Lowenstein CJ, Snyder SH, and Russell SW (1993) Expression of nitric oxide synthase gene in mouse macrophages activated for tumor cell killing. J Biol Chem 268:1908-1913.

Maier-Hauff K, Baethmann A, Lange M, Schürer L, and Unterberg A (1984). The kallikrein-kinin system as mediator in vasogenic brain edema. Part 2: Studies on kinin formation in focal and perifocal brain tissue. J Neurosurg 61:97-106.

Mayhan WG (1995). Role of nitric oxide in disruption of the blood-brain barrier during acute hypertension. Brain Res 686:99-103.

Murohara T, Asahara T, Silver M, Sauters C, Masuda H, Kalka C, Kearney M, Chen D, Symes JF, Fishman MC, Huang PL, and Isner JM (1998). Nitric oxide synthase modulates angiogenesis in response to tissue ischemia. J Clin Invest 101: 2567-2578.

Nag S (1996). Cold-injury of the cerebral cortex: Immunolocalization of cellular proteins and blood-brain barrier permeability studies. J Neuropathol Exp Neurol 55:880-888.

Nag S, Takahashi JL, and Kilty DW (1997). Role of vascular endothelial growth factor in blood-brain barrier breakdown and angiogenesis in brain trauma. J Neuropathol Exp Neurol 56:912-921.
Nathan C (1992). Nitric oxide as a secretory product of mammalian cells. FASEB J 6:3051-3064.

Olson JJ, Beck DW, Warner DS, and Coester H (1987). The role of new vessels and macrophages in the development and resolution of edema following a cortical freeze lesion in the mouse. J Neuropathol Exp Neurol 46:682-694.

Papapetropoulos A, Desai KM, Rudic RD, Mayer B, Zhang R, Ruiz-Torres MP, Garcia-Cardena G, Madri JA, and Sessa WC (1997). Nitric oxide synthase inhibitors attenuate transforming-growth-factor- $\beta 1$-stimulated capillary organization in vitro. Am J Pathol 150:1835-1844.

Picard P, Smith PJW, Monge JC, Rouleau JL, Nguyen QT, Calderone A, and Stewart DJ (1998). Coordinated upregulation of the cardiac endothelin system in a rat model of heart failure. J Cardiovasc Pharmacol 31(Suppl 1):S294-S297.

Rudic RD, Shesely EG, Maeda N, Smithies O, Segal SS, and Sessa WC (1998). Direct evidence for the importance of endothelium-derived nitric oxide in vascular remodeling. J Clin Invest 101:731-736.

Stanarius A, Seidel B, and Wolf G (1998). Neuronal nitric oxide synthase in the vasculature of the rat brain: An immunocytochemical study using tyramide signal amplification. J Neurocytol 27:731-736.

Torreilles F, Salman-Tabcheh S, Guérin M-C, and Torreilles J (1999). Neurodegenerative disorders: The role of peroxynitrite. Brain Res Rev 30:153-163.

van der Veen RC, Hinton DR, Incardonna F, and Hofman FM (1997). Extensive peroxynitrite activity during progressive stages of central nervous system inflammation. J Neuroimmunol 77:1-7.

Wada K, Chatzipanteli K, Kraydieh S, Busto R, and Dietrich WD (1998). Inducible nitric oxide synthase expression after traumatic brain injury and neuroprotection with aminoguanidine treatment in rats. Neurosurgery 43:1427-1436.

Xie QW and Nathan C (1994). The high-output nitric oxide pathway: Role and regulation. J Leukocyte Biol 56:576-582.

Ziche M, Morbidelli L, Choudhuri R, Zhang HT, Donnini S, and Granger HJ (1997). Nitric oxide synthase lies downstream from vascular endothelial growth factor-induced but not basic fibroblast growth factor-induced angiogenesis. J Clin Invest 99:2625-2634. 